\title{
Más allá de la casa sin nombre. Nuevos estudios: Arahal
}

\section{Vidal Gómez Martínez ${ }^{a}$, Blanca del Espino Hidalgo ${ }^{b}$, María Teresa Pérez Canoc}

${ }^{a}$ Universidad de Sevilla, Avenida de la Reina Mercedes 2, 41012 Sevilla, vidalgomezmartinez@gmail.com, ${ }^{b}$ Instituto Andaluz del Patrimonio Histórico, Camino de los Descubrimientos s/n, 41092 Sevilla, blanca.espino@juntadeandalucia.es, Universidad de Sevilla, Avenida de la Reina Mercedes 2,41012 Sevilla, tpcano@us.es

\begin{abstract}
Resumen
Frente a los estudios generalizados de la arquitectura singular, de autor o con un amplio reconocimiento social, investigaciones sobre temas como la casa tradicional han sido más minoritarias. Trabajos realizados por los autores en los últimos años han permitido identificar y caracterizar una tipología de casa popular que se encontraba hasta entonces englobada en tipologías genéricas con las que comparte ciertos elementos pero en la que tienen cabida casas de diversa índole. Se trata de una casa asociada a los crecimientos y densificaciones de los tejidos urbanos medievales durante la época moderna en la provincia de Sevilla y territorios limitrofes, llegando en plenitud de uso hasta la primera mitad del siglo XX cuando cae en desuso por los cambios tecnológicos en el ámbito agropecuario. Esta casa ocupa parcelas de poco frente y mucho fondo, conformándose mediante cuerpos - normalmente de doble crujía - paralelos a fachada que se alternan con espacios libres interiores, mediante la secuencia tipológica constituida por un cuerpo de fachada, un patio, un cuerpo intermedio y un corral de fondo. Todo el conjunto se registra mediante un sistema de pasos independientes - zaguán y paso intermedio - que salvan las estancias de servidumbres de paso para permitir el acceso de animales y aperos de forma limpia hasta el corral.
\end{abstract}

Tras el trabajo inicial titulado "La casa sin nombre. Una casa popular tradicional en la provincia de Sevilla" se han continuado los trabajos de investigación ampliando el ámbito de estudio y actualizando fuentes y referencias a partir del mismo procedimiento metodológico basado en el reconocimiento de esta arquitectura a través de publicaciones especializadas y sobre todo en el análisis de los contenidos de los Planes Especiales de Protección de los Conjuntos Históricos mediante un proceso de parametrización común de la información de los diferente planes.

Concretamente, este trabajo se centra en el análisis del Plan Especial de Protección del Conjunto Histórico de Arahal, municipio de la provincia de Sevilla con un amplísimo caserío caracterizado por la conservación de su arquitectura popular doméstica donde se localiza claramente esta casa popular de zaguán, patio y corral.

Palabras clave: casa popular, arquitectura vernácula, arquitectura tradicional, Arahal, conjunto histórico.

\begin{abstract}
In contrast to the generalized studies of singular or signature architecture with wide social recognition, investigations on subjects such as the traditional house have been more of a minority. Previous works carried out by the authors in recent years have made it possible to identify and characterize a popular house typology which up until then had been included in generic typologies with which it shares certain elements but in which houses of different types have a place. It is a house associated with the growth and densification of medieval urban fabric during the modern period in the province of Seville and neighbouring territories, reaching its full use until the first half of the 20th century when it fell into disuse due to technological changes in the agricultural field. This house occupies plots of land with little front and a lot of depth, being formed by bodies - normally with a double bay - parallel to the façade that alternate with free interior spaces, by means of the typological sequence constituted by a façade body, a courtyard, an intermediate body and a back yard. The whole complex is registered by means of a system
\end{abstract}


of independent passages - hallway and intermediate passage - that save the rooms of servitude of step to allow the access of animals and tools cleanly up to the corral.

After the initial work entitled "The house without a name. A traditional popular house in the province of Seville", research work has continued to expand the scope of the study and update sources and references based on the same methodological procedure based on the recognition of this architecture through specialized publications and above all on the analysis of the contents of the Special Plans for the Protection of Historic Areas through a process of common parameterization of the information in the different plans.

Specifically, this work focuses on the analysis of the Special Plan for the Protection of the Historical Centre of Arahal, a municipality in the province of Seville with a very large housing set characterized by the conservation of its popular vernacular domestic architecture where this popular house with a hallway, patio and backyard is clearly located.

Keywords: popular house, vernacular architecture, traditional architecture, Seville, historical site. 


\section{Introducción}

\subsection{La casa sin nombre}

La línea de investigación que continua este trabajo ha permitido identificar, localizar y caracterizar una casa popular tradicional que hasta este momento permanecía englobada en la amalgama de denominaciones - a menudo más vinculadas a la posición social de sus moradores que a sus propias características arquitectónicas - y encuadres tipológicos con los que se identifica la arquitectura popular doméstica sevillana, tanto en los estudios de escala general - nacional o regional - como en los de escala local. Una casa que no tenía un reconocimiento cultural propio y que por no tener no tenía ni nombre.

Se trata de una casa entre medianeras asociada al parcelario de origen medieval y propio de los crecimientos de época moderna en Andalucía Occidental - fundamentalmente la provincia de Sevilla y territorios limítrofes - basado en grandes manzanas que generan parcelas de poco frente y mucho fondo. En los casos analizados anteriormente, las dimensiones de las parcelas se sitúan mayoritariamente entre los 6 y 12 metros de frente y entre los 20 y los 40 metros de fondo, con unas superficies que suelen oscilar entre los 100 y los 300 metros cuadrados.

Se configura a través de un mecanismo de yuxtaposición de cuerpos construidos alternados con espacios libres, según una secuencia tipológica clara definida por la presencia de un cuerpo de fachada, un patio, un cuerpo intermedio y un corral de fondo. Toda la estructura se registra mediante un sistema de pasos independientes constituido por el zaguán de acceso y el paso del cuerpo intermedio, que salvan las estancias vivideras de servidumbres de paso, permitiendo el acceso de animales y aperos de labranza hasta el corral - elementos propios de los habitantes de estas casas que trabajaban la tierra con medios propios y necesitaban espacio para el almacenamiento de los utensilios, así como del resultado de las cosechas - . Los cuerpos construidos son generalmente de doble crujía, permitiendo la ventilación directa de las estancias bien hacia la calle o bien hacia los espacios libres interiores y cuentan con una altura de una planta y soberado - espacio bajo cubierta destinado originalmente al almacenamiento de utensilios y cosecha que paulatinamente ha ganado altura para albergar usos vivideros - . En el patio se localizan a menudo estructuras auxiliares, como la cocina o la escalera de acceso al nivel superior, donde en ocasiones se desarrolla una galería lateral para mejorar el acceso. Por la introducción de espacios libres interiores y la limitación de las alturas, genera un caserío de una densidad edificatoria baja, mayoritariamente inferior a 1,25 metros cuadrados de techo por cada metro cuadrado de parcela.
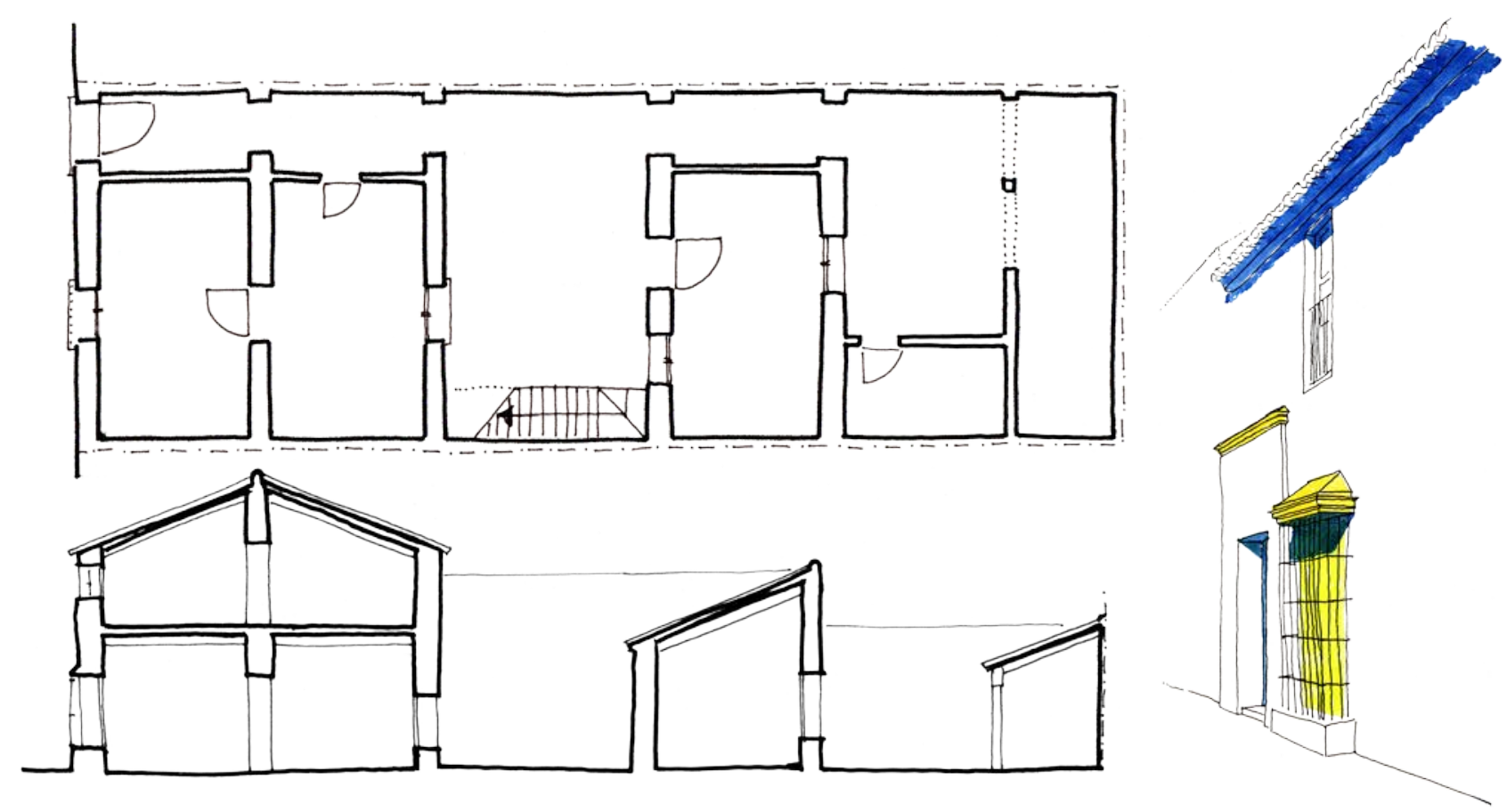

Fuente: Gómez Martínez, V (2017)

Fig. 1 Esquema tipológico y vista de una casa en Lebrija 
Se basa en sistemas constructivos sencillos, ligados a los recursos locales y a las limitaciones económicas. Los muros son de tapial, ladrillo e incluso mampuesto de entre 40 y 70 centímetros de espesor, mientras que los forjados son de rollizos o escuadrías de madera con alfajías de madera o de ladrillo por tabla, con luces que oscilan entre los 2,5 y los 3 metros. Tanto los forjados de planta como los de cubierta se ejecutan de este modo, apoyándose estos últimos directamente sobre los muros peraltados o bien sobre cerchas de par y nudillo que montan sobre los muros exteriores. Las cubiertas son de teja árabe, aunque han sufrido un fuerte proceso de sustitución - principalmente en los cuerpos interiores - tanto en sus elementos de finalización - en el que las chapas de acero y fibrocemento han sustituido a las tejas - como en el propio sistema constructivo al sustituirse por cubiertas planas.

$\mathrm{Su}$ formalización es sencilla, basada en el blanco omnipresente de las paredes encaladas. Únicamente reciben cierto ornamento las portadas con resalte y listón superior - de claro origen mudéjar - , las ventanas en saledizo y en ocasiones zócalos a la tirolesa encalados en gris o albero. A lo largo de su extensa cronología - que comienza con la estabilización posterior a la reconquista a partir del siglo XIV y finaliza con el descarte de esta tipología ligado a la industrialización tanto de la agricultura como de la edificación en la primera mitad del siglo XX - , ha conservado su sencillez característica, si bien las portadas han recibido un cierto proceso de barroquización y durante las últimas décadas ha recibido una clara influencia regionalista (Fig. 1).

Conforme a sus características tipológicas, finalmente se identificó como casa popular de zaguán, patio y corral (Gómez, 2017).

\subsection{Metodología}

Este estudio inicial se planteó con una metodología clara basada en tres líneas de trabajo.

- Trabajos previos fundamentados en la investigación bibliográfica y archivística, con el objetivo de realizar un primer esbozo de una tipología reconocida de forma directa, pero que no contaba con un registro documental. Concretamente, se perseguía la realización de un esquema tipológico inicial, un reconocimiento territorial aproximado y un encuadre histórico general que permitiera acotar el objeto y el ámbito de estudio, así como establecer tres casos de estudio válidos: Carmona, Cazalla de la Sierra y Lebrija - por recoger cada una de las unidades geográficas reconocibles en el ámbito de estudio y contar con Planes Especiales de Protección de sus Conjuntos Histórico con una alta resolución y amplitud - .

- Caracterización de la tipología identificada a partir del análisis de la documentación contenida en los Planes Especiales de Protección de los Conjuntos Históricos seleccionados como casos de estudio, realizando una base de datos que unificara los criterios de caracterización dispares de cada Plan para permitir una lectura conjunta de los mismos. La lectura de la base de datos permitió extraer la síntesis de los elementos que caracterizan esta tipología y su ubicación planimétrica permitió su encuadre cronológico en los procesos de evolución urbana.

- Contraste de resultados mediante la realización de publicaciones parciales y presentaciones en encuentros internacionales sobre patrimonio y arquitectura popular.

\subsection{Nuevos objetivos}

Ya en el estudio inicial se planteaban candidatos claros para la continuación de la investigación - que no tuvieron cabida en el mismo por las limitaciones propias del formato - como es el caso de los Conjuntos Históricos de Arahal, Marchena o Utrera. Al mismo tiempo se establecía que para la correcta identificación de una tipología de arquitectura popular era necesaria la lectura sintética de los trabajos locales que, aunque ofrecieran resultados precisos, carecían de una visión de la misma en su verdadera magnitud cultural en cuanto a implantación territorial, cronológica y social.

Como se ha referido, se trata de una línea de investigación activa. En este sentido, la continuación de la investigación bibliográfica sostenida ha permitido localizar ejemplos de esta casa en el sur de la provincia de Badajoz (Martín, 2004) y en el noroeste de la provincia de Córdoba (Mendoza, 2009). Al mismo tiempo se desarrollan trabajos cuyos objetivos 
fundamentales son la revisión sistemática de la metodología anteriormente descrita y la identificación y caracterización de la casa de zaguán, patio y corral en nuevos Conjuntos Históricos, en este caso concreto, el de Arahal.

\section{La arquitectura popular doméstica en Arahal}

Las dimensiones de la zona histórica del núcleo, el estado de conservación del caserío y su uniformidad ya resaltados en otros trabajos de los autores (Gómez, 2007) hacen de Arahal un ámbito de estudio perfecto para la arquitectura popular doméstica (Gómez et al., 2008). Sin embargo, la arquitectura popular de Arahal ha pasado desapercibida para los grandes trabajos de referencia en este campo, tanto de escala nacional como regional y desde los primeros hasta los más recientes.

Ni las grandes obras de arquitectura popular en España de los años treinta de Fernando García Mercadal (García, 1930) o Leopoldo Torres Balbás (Torres, 1930) ni los recorridos más detallados por la geografía nacional realizados posteriormente por Carlos Flores (Flores, 1973), José Claret Rubira (Claret, 1976) o Luis Feduchi (Feduchi, 1798), hacen referencia expresa alguna a la arquitectura popular en Arahal. Tampoco reparan en Arahal la frustrada segunda fase del Inventario andaluz de arquitectura popular (Melero, 1995) ni trabajos de escala provincial con mayor resolución como los realizados por Juan Agudo Torrico (Agudo, 1984) o Salvador Rodríguez Becerra (Rodríguez, 2001).

Esta circunstancia no es sino un claro reflejo de las dificultades metodológicas a las que se enfrenta el estudio de la arquitectura popular, que necesita del concurso de equipos transdisciplinares y estudio de diversas escalas para obtener una visión completa de este Patrimonio Cultural. De hecho, ni tan siquiera las diferentes Leyes de Patrimonio - tanto estatales como regionales - han establecido una categoría propia de protección más allá de la figura del Conjunto Histórico, que recoge sus valores de conformación de la trama y el ambiente urbanos como conjunto, dejando a un lado los valores propios de cada unos de los elementos que lo componen, como arquitectura definitoria y soporte de su cultura.

Por todo ello, las figuras del Conjunto Histórico y del Plan Especial de Protección adquieren un papel relevante en el estudio, protección y conservación de la arquitectura popular, ya que son los únicos trabajos que afrontan un estudio de la misma con equipos transdisciplinares - generalmente compuestos por arquitectos, urbanistas, historiadores, arqueólogos y antropólogos - y con la máxima resolución, aunque estén limitados a la escala local por la propia definición del concepto. De ahí que una lectura sintética de los Planes Especiales sea esencial para percibir la verdadera dimensión cultural de la arquitectura popular, superando la escala local.

\subsection{El Conjunto Histórico}

El centro histórico de Arahal cuenta con una temprana declaración como Conjunto Histórico en 1979 - en base a la ley de Patrimonio de 1933 - en la que se establecen tres "zonas artísticas" y una "zona de respeto", sin desarrollarse los valores que justifican su declaración. Posteriormente, en 2003 se establece una delimitación unitaria del Conjunto Histórico (Fernández, 2017) - que se ajusta a la extensión del núcleo urbano recogido en el levantamiento del Instituto Cartográfico y Estadístico de 1896 - , incluyéndose una amplia justificación de los valores patrimoniales del Conjunto Histórico donde destaca la presencia y relevancia del caserío vernáculo, tanto por la conservación de la trama como de las tipologías edificatorias.

"El Conjunto Histórico está compuesto por un importante número de viviendas de crujías paralelas a fachada con patio posterior, que conforman un parcelario histórico de gran homogeneidad y destacados valores patrimoniales. Esta tipología de viviendas es exponente de la estructura social histórica que ha existido en esta población que, durante siglos, ha configurado tanto la morfología de sus edificaciones como las sucesivas ampliaciones urbanísticas y los espacios públicos con que cuenta. Conservada aún en gran medida, está muy vinculada a la explotación agraria, (...) que ha propiciado la creación de infraestructuras para su transformación y almacenamiento".

Es la primera referencia clara en el ámbito patrimonial a la arquitectura popular doméstica de Arahal y hace alusión directa al proceso de configuración tipológica de la casa de zaguán patio y corral. Cabe resaltar que las primeras 
noticias de Arahal como asentamiento urbano aparecen tras la conquista del Reino de Sevilla por parte de la Corona de Castilla y la cesión de los terrenos a la orden de Alcántara, por lo que el desarrollo urbano de Arahal coincide rigurosamente con la cronología establecida para la casa de zaguán, patio y corral (Gómez, 2017), lo que parece explicar su presencia masiva probablemente por su encaje en una trama urbana que surge al mismo tiempo y con los mismos requerimientos sociales, culturales y económicos.

\subsection{El Plan Especial de Protección}

El Plan Especial de Protección del Conjunto Histórico de Arahal (PEPCHA) elaborado por la Delegación de Urbanismo del Ayuntamiento de Arahal - bajo la dirección de los arquitectos municipales Rafael González Millán y José Julio Ortega Godino - y aprobado inicialmente en 2006, es un amplio y profundo documento de análisis y reconocimiento del Patrimonio Histórico de la localidad. Hace especial hincapié en la extensión, homogeneidad y conservación del caserío, que se identifica como uno de los principales valores y señas de identidad del Conjunto Histórico.

\subsubsection{Tipologías de arquitectura popular doméstica}

El documento expone un análisis de fundamentación claramente arquitectónica que identifica un preciso proceso de configuración tipológica del caserío en base a la disposición de cuerpos construidos de crujías paralelas a fachada que se alternan con espacios libres. Más en profundidad, establece tres tipologías de arquitectura popular doméstica - a la que se suman dos de casa señorial que queda fuera del ámbito de estudio del presente trabajo - con criterios de base socio-antropológica e histórica.

- Casa popular de crujías yuxtapuestas. Se corresponde con el tipo más modesto, compuesto por un cuerpo de fachada de doble crujía y un espacio libre posterior, debido a las limitaciones de la parcela -hasta $250 \mathrm{~m}^{2}$ y $6 \mathrm{~m}$ de frente - .

- Casa de labor de la pequeña burguesía agraria. Se trata de casas de mayor envergadura - en parcelas de hasta $600 \mathrm{~m}^{2}$ y $12 \mathrm{~m}$ de frente - que se desarrolla con un cuerpo de fachada, patio, cuerpo intermedio y corral. En los espacios libres se ubican construcciones auxiliares y galerías de paso.

- Casa historicista de la pequeña burguesía agraria. Con dimensiones y disposición similares a la casa de labor, la casa historicista se identifica con el empleo de esta corriente arquitectónica como rasgo de distinción social.

\subsubsection{Catalogación}

Al extenso Conjunto Histórico - de 2.170 parcelas catastrales - le corresponde un amplio catálogo de inmueble que recoge 333 fichas (15,35\%) correspondientes a inmuebles catalogado A, B, C y D y 1.260 inmuebles $(58,06 \%)$ catalogados $\mathrm{E}$ - sin ficha - . Se trata de fichas de una sola página que recogen, además de los datos de identificación y localización del inmueble, una descripción literal del mismo - donde se refleja la tipología, distribución y sistemas constructivos - , planimetría catastral de cubiertas a 1/1.000, ortofotografía y fotografías del inmueble - de fachada y de un detalle - .

\subsubsection{Protección}

El PEPCHA establece cinco niveles de protección de los inmuebles - A integral, B tipológico, C parcial, D estructural y E ambiental- en función de sus valores patrimoniales - históricos, arquitectónicos, urbanos, visuales, morfológicos y etnológicos - y que permiten paulatinamente un grado de intervención mayor en los inmuebles. Tanto las intervenciones permitidas como las zonas de protección se especifican literal y gráficamente en las fichas de catálogo, siendo predominantemente una protección puntual sobre el cuerpo construido de fachada $(93,53 \%)$ y en el mejor de los casos realizando una protección parcial que excluye el corral (6,47\%). Los numerosos ejemplos de la arquitectura popular doméstica - casas popular, de labor e historicista - cuentan con niveles de protección de B, C, D y E. El presente trabajo toma como casos de estudio los 299 inmuebles con protección B, C y D que cuentan con ficha de catálogo, ya que de los inmuebles catalogados $\mathrm{E}$ no se dispone de la información necesaria al carecer de ficha de catálogo (Tabla 1). 
Tabla 1. Catalogación de la arquitectura popular doméstica en el PEPCHA

\begin{tabular}{lccccc} 
Tipologías $\backslash$ protección & B & C & D & total & \% \\
\hline Casa popular & 0 & 6 & 98 & 104 & 33,23 \\
Casa de labor & 13 & 50 & 73 & 136 & 40,84 \\
Casa historicista & 12 & 44 & 3 & 59 & 17,72 \\
\hline Total & $\mathbf{2 5}$ & $\mathbf{1 0 0}$ & $\mathbf{1 7 4}$ & $\mathbf{2 9 9}$ & $\mathbf{8 9 , 7 9}$
\end{tabular}

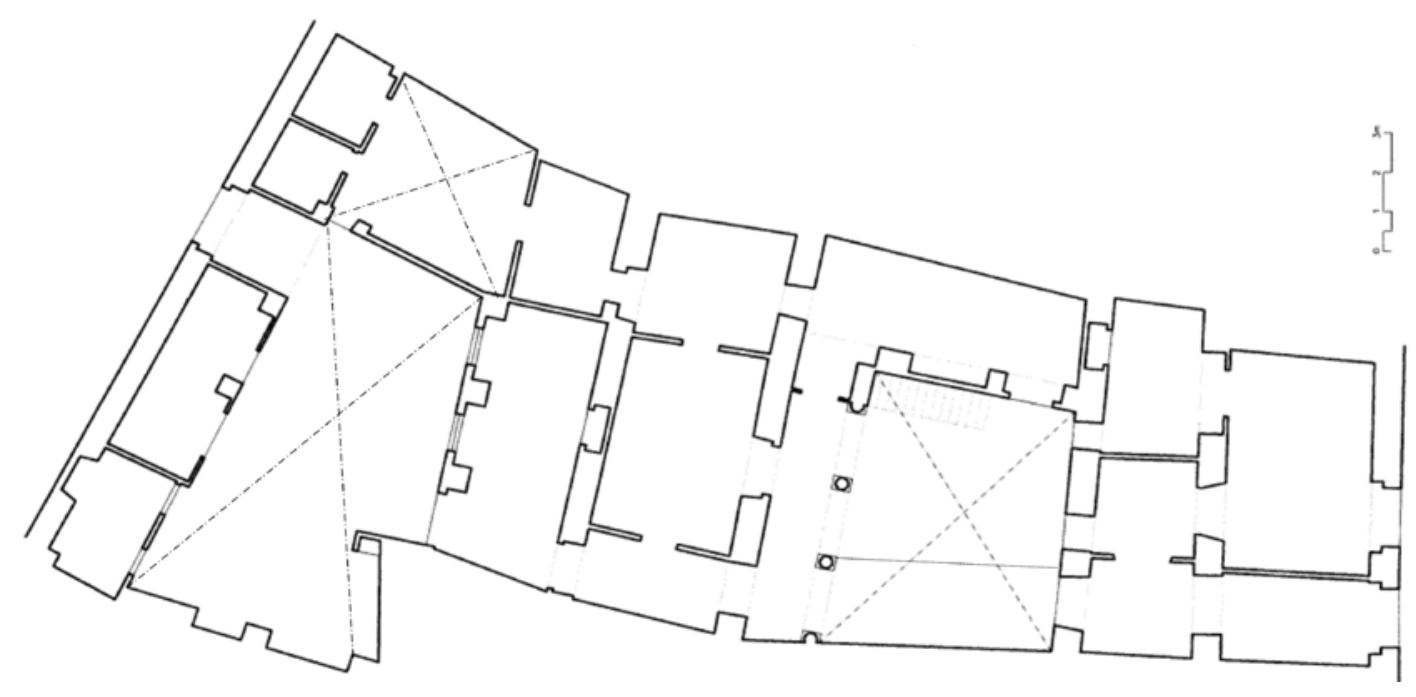

Fuente: Plan Especial de Protección del Conjunto Histórico de Arahal. Memoria de información.

Fig. 2 Casa de labor en la calle Marchena 6
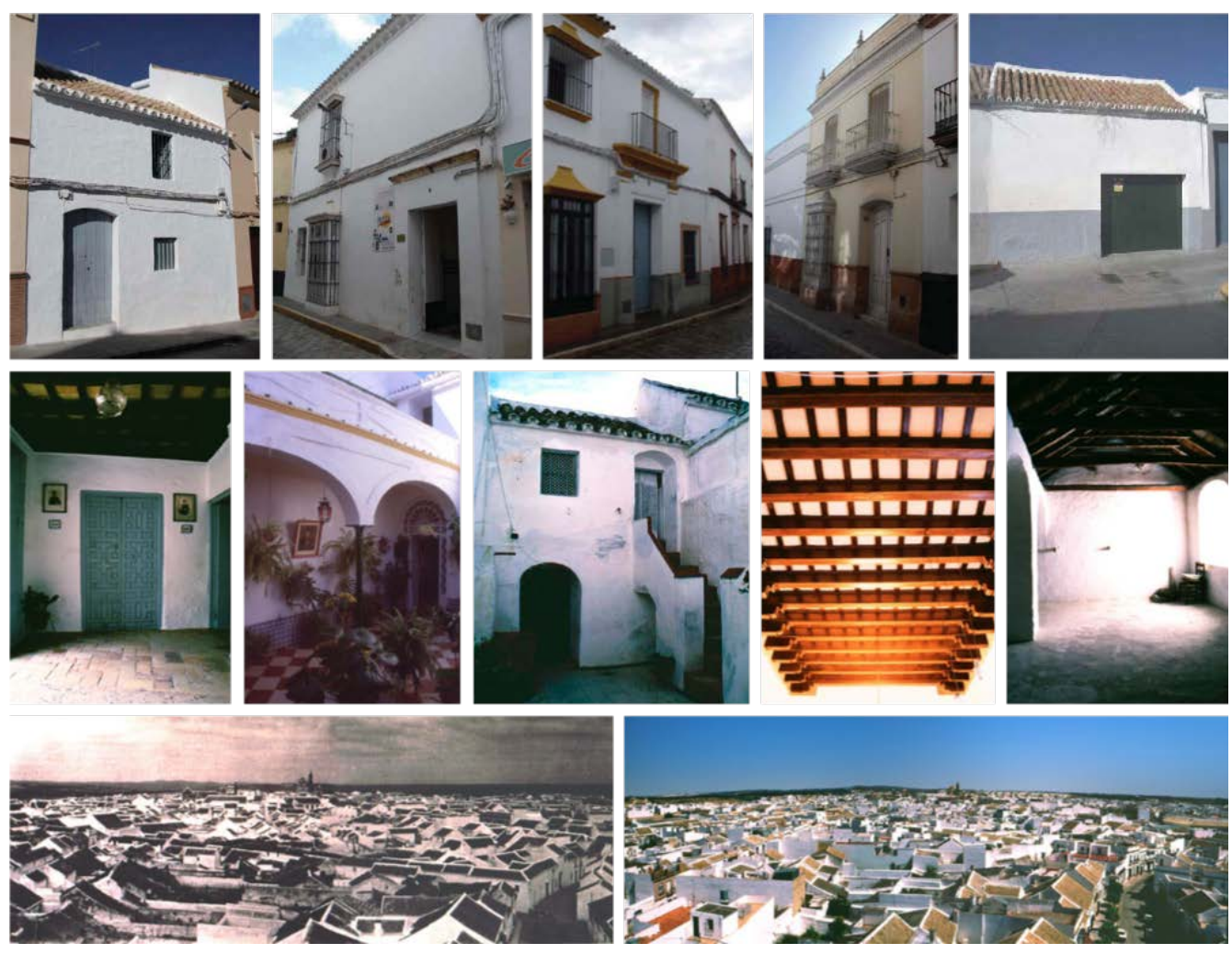

Fuente: Plan Especial de Protección del Conjunto Histórico de Arahal.

Fig. 3 Caracterización de la arquitectura popular doméstica en Arahal 


\section{La casa de zaguán, patio y corral en Arahal \\ 3.1. Encuadre tipológico}

De la descripción de las tipologías mencionadas en el apartado anterior se deduce una clara diferenciación entre las mismas. La popular de crujías yuxtapuestas responde a una estructura más sencilla, mientras que la casa de labor y la casa historicista comparten una estructura más compleja y se diferencian entre sí por la formalización y la calidad de los elementos constructivos. Sin embargo, analizadas a través de las plantas y descripciones incluidas en las fichas, se aprecia un esquema de conformación tipológica mucho más unitario, compuesto por un cuerpo de fachada de doble crujía, un patio, un cuerpo intermedio normalmente de doble crujía y un corral trasero (Fig. 2).

Este esquema tipológico se corresponde con exactitud al de la casa de zaguán, patio y corral, tipología en la que pueden englobarse estos tres tipos identificados por el PEPCHA, quedando al margen los inmuebles con una configuración extremadamente sencilla o compleja para esta tipología. La caracterización formal de los inmuebles tiene una relevancia secundaria en la identificación tipológica y la presencia de inmuebles de formalización de tradición mudéjar, barroca o historicista - tomando la terminología del PEPCHA - dentro de una misma tipología es común en tipos con cronologías dilatadas como es el caso de la casa de zaguán, patio y corral. De hecho, esta casa de zaguán, patio y corral tiene una amplia presencia en el Conjunto Histórico de Arahal - como se recoge en la tabla 2 en relación a los inmuebles con ficha de catálogo que son los tipos A, B, C y D - , fundamentalmente entre las casas popular y de labor, superando el $58 \%$ de lo inmuebles de esta última tipología.

Tabla 2. La casa de zaguán, patio y corral en el catálogo del PEPCHA

\begin{tabular}{lccccccc} 
& & \multicolumn{6}{c}{ Casa de zaguán, patio y corral } \\
\cline { 3 - 8 } tipologías PEPCHAlprotección & & A & B & C & D & total & \% \\
\hline Casa popular & 104 & 0 & 0 & 5 & 44 & $\mathbf{4 9}$ & $\mathbf{4 7 , 1 2}$ \\
Casa de labor & 136 & 0 & 8 & 31 & 40 & $\mathbf{7 9}$ & $\mathbf{5 8 , 0 9}$ \\
Casa historicista & 59 & 0 & 2 & 9 & 0 & $\mathbf{1 1}$ & $\mathbf{1 8 , 6 4}$ \\
\hline total & $\mathbf{2 9 9}$ & $\mathbf{0}$ & $\mathbf{1 0}$ & $\mathbf{4 5}$ & $\mathbf{8 4}$ & $\mathbf{1 3 9}$ & $\mathbf{4 6 , 4 9}$
\end{tabular}

\subsection{Caracterización}

\subsubsection{Configuración tipológica y arquitectónica}

Tanto las descripciones de las tipologías analizadas en la memoria de información del PEPCHA como las notas incluidas en las fichas de catálogo, hacen un retrato preciso de estos inmuebles que se ajusta con claridad al de la casa de zaguán, patio y corral. Esta identificación se produce tanto a nivel de desarrollo tipológico como de sistemas constructivos y formalización (Fig. 3).

A nivel tipológico se repite sistemáticamente la alternancia de cuerpos construidos - mayoritariamente de doble crujía $(87,05 \%)$ con planta baja y soberado $(69,78 \%)$ - con espacios libres, siguiendo la secuencia de cuerpo de fachada, patio, cuerpo intermedio y corral, quedando registrado todo el conjunto por el acceso independiente a través del zaguán [figura 3f] y el paso intermedio hasta el corral. En el patio principal se localizan construcciones complementarias, escaleras exteriores de acceso a los soberados y galerías [figura $3 \mathrm{~g}$ y $3 \mathrm{~h}$ ], a menudo mediante arcadas, mientras que en el corral se ubican construcciones auxiliares contando en muchas ocasiones con un acceso propio por una calle diferente [figura 3e].

A nivel constructivo, se trata de inmuebles de muros de ladrillo o tapial, sobre los que apoyan forjados de escuadría de madera - o rollizos - con alfajía de ladrillo por tabla [figura 3i]. Las cubiertas son mayoritariamente de teja árabe $(92,81 \%)$ apoyada sobre forjados inclinados de muro a muro o bien mediante cerchas de par y nudillo [figura $3 \mathrm{j}]$, con una gran presencia en el paisaje urbano [figura $3 \mathrm{k}$ y 31 ].

La formalización de los inmuebles refleja la adaptación de la tipología a diferentes épocas desde las portadas en resalto propias de la herencia mudéjar $(18,71 \%)$ a las más recientes de carácter historicista $(10,07 \%)$, pasando por los 
inmuebles de formalización más barroca [figura 3a a 3d] propias de los siglos XVII y XVIII (13,67\%), aunque la solución predominante es la más sencilla, carente de todo ornamento $(57,55 \%)$.

Los parámetros dimensionales de los inmuebles nos muestran un predominio claro de parcelas pequeñas y medianas con superficies entre 100 y $300 \mathrm{~m}^{2}$ (71,94\%) con frentes de fachada por debajo de los 8 metros $(76,98 \%)$ y una densidad edificatoria baja, mayoritariamente por debajo del $1,25 \mathrm{~m}^{2} / \mathrm{m}^{2}(66,91 \%)$.

\subsubsection{Implantación urbana y cronología}

Si bien las dimensiones principales de estos inmuebles analizadas en el apartado anterior siguen los parámetros observados en los estudios anteriores, el análisis de la relación de la tipología con la trama urbana ofrece datos significativos (Fig. 4).

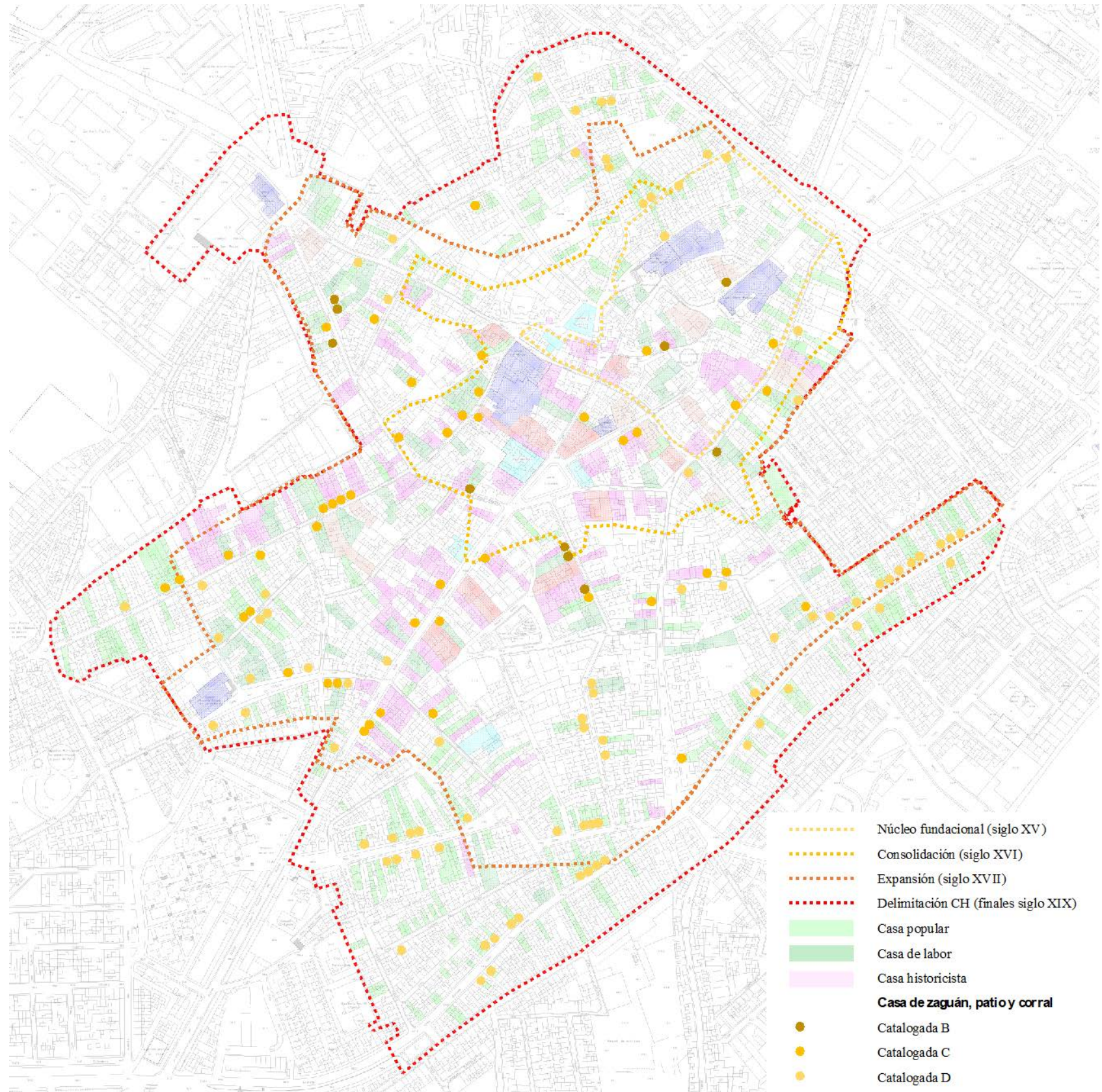

Fuente: Elaborado por los autores sobre la base planimétrica del PEPCHA.

Fig. 4 La casa popular de zaguán, patio y corral en Arahal 1/6.000 
Por un lado, se observa una clara disposición de los inmuebles a lo largo de las grandes vías de entrada y salida de la localidad como pueden ser las calles Dr. Gamero, Corredera-Puerta de Utrera, Barriete o Sevilla, que discurren sobre las antiguas veredas que comunicaban Arahal con Sevilla, Utrera, Villamartín o Morón.

Por otro lado, gran número de los inmuebles se encuentran en parcelas pasantes - con frente a dos calles, excluidas las parcelas en esquina - con independencia del fondo de las mismas, concretamente 48 de los 139 inmuebles considerados (34,53\%). La recuperación de los datos de los estudios realizados en Carmona, Cazalla de la Sierra y Lebrija ha permitido contrastar que en Cazalla de la Sierra el fenómeno ofrece proporciones similares, concretamente 15 de los 40 inmuebles considerados $(34,53 \%)$, mientras que el Carmona y Lebrija se trata de una configuración casi residual, concretamente 16 de los 164 inmuebles considerados (9,75\%) en Carmona y 6 de los 39 inmuebles considerados $(15,38 \%)$ en Lebrija. Esto se debe a que mientras Carmona y Lebrija son núcleos urbanos que crecen a partir de tejidos medievales y romanos caracterizados por manzanas de gran fondo, Cazalla de la Sierra - que en época árabe se limitaba a una pequeña fortificación - y Arahal responden a crecimientos que se desarrollan ya en época cristiana - generados a partir de manzanas de forma alargada - y demuestra la relevancia del tipo estudiado tanto en su aspecto arquitectónico como en su implicación en la conformación de la ciudad. El PEPCHA hace una pertinente protección del parcelario como elemento profundamente ligado a la arquitectura - permitiendo la segregación en sólo 2 de los 139 inmuebles, debido a que su configuración actual responde a procesos de agregación que distorsionan el carácter originario de los inmuebles.

La datación de los inmuebles recogida en las fichas de catálogo es parcial y ofrece datos contradictorios. 37 de los 139 inmuebles carecen de datación alguna $(26,62 \%)$, mientras que entre los inmuebles datados solo se localiza 1 del siglo XIX, siendo los otros 101 del siglo XX, con dataciones que llegan hasta final de los años sesenta. Estos datos ofrecen una datación contradictoria por dos motivos. Por un lado, se reconocen elementos arquitectónicos con una formalización propia de los siglos XVII y XVIII y por otro, esta datación generalizada de inmuebles en el siglo XX se correspondería con unos procesos de sustitución sistemática del caserío entre los años veinte y sesenta de los que no se tienen noticias y a los que el propio plan no hace referencia alguna en su amplia descripción de la evolución urbana de la ciudad. Esta datación solo puede explicarse mediante el empleo de referencias exclusivamente registrales y catastrales - donde a menudo se sitúa el origen de la edificación en el momento de su registro y no de su construcción así como la falta de participación de historiadores y arqueólogos en el equipo de redacción.

\section{Conclusiones}

De forma sucinta, en este trabajo pueden extraerse las siguientes conclusiones.

La ampliación del trabajo de reconocimiento de la casa de zaguán, con Arahal como nuevo caso de estudio, confirma la validez de la metodología empleada a pesar de las diferencias naturales entre los diferentes planes especiales de protección y de la falta de elementos como la planimetría interior - dificultando, pero no impidiendo la lectura tipológica - , que han permitido que se pueda cumplimentar la base de datos con nuevas fichas con más de $2 / 3$ de los campos completados.

La obtención y comparación de nuevos datos permite perfilar con mayor precisión las características, implantación territorial y cronología de esta casa de zaguán, patio y corral. Se asocia claramente a los procesos de crecimiento y consolidación de época moderna, encontrándose en mayor número en ciudades sin tejidos medievales anteriores como Cazalla de la Sierra y Arahal. Además, en estas ciudades se identifica una mayor implicación entre tipología y morfología, evidenciada por la presencia representativa de parcelas con doble frente que facilitan el acceso al corral. Igualmente se localiza una mayor presencia de estos inmuebles en las ciudades de la campiña - Carmona y Arahal frente a las de otras unidades territoriales.

El PEPCHA goza de una visión con una amplia perspectiva desde el ámbito arquitectónico, pero ofrece resultados contradictorios en la datación de los inmuebles que impiden realizar un correcto encuadre cronológico de la casa de zaguán, patio y corral en Arahal como se ha realizado en estudios anteriores. 
Por último, se pone de manifiesto la necesidad de abordar el estudio de la arquitectura popular a partir de estudios transdisciplinares, compuestos por diferentes profesionales que aporten el rigor y la perspectiva de cada una de sus disciplinas, evitando resultados parciales y lecturas incorrectas de ciertos elementos. Así, resulta imprescindible la presencia de arquitectos que analicen la configuración arquitectónica y constructiva, arqueólogos e historiadores que definan con precisión la cronología de los inmuebles mediante la comparación de elementos formales y sistemas constructivos y antropólogos que recojan la tradición de uso de estos inmuebles, mientras perdure su memoria.

\section{Nota}

Nota final: este trabajo ha sido elaborado de forma conjunta por los autores, correspondiendo la redacción del cap. 1 a María Teresa Pérez Cano y Blanca del Espino Hidalgo y la de los capítulos 2, 3 y 4 a Vidal Gómez Martínez.

\section{Referencias}

Agudo Torrico, J. (1984). Arquitectura popular en la provincia de Sevilla. En Sevilla y su provincia. Sevilla: Ediciones GERVER SL. Claret Rubira, J. (1976). Detalles de arquitectura popular española. Barcelona: Gustavo Gili.

Feduchi, L., Borrego, F., y Temprano, J. (1978). Itinerarios de arquitectura popular española. Barcelona: Editorial Blume.

Fernández Ruiz, D. (2017). Análisis de la normativa y la protección del municipio de Arahal (Sevilla). Trabajo de Curso. Sevilla: Universidad de Sevilla.

Flores, C. (1973). Arquitectura popular española. Madrid: Aguilar SA de ediciones.

García Mercadal, F. (1930). La casa popular en España. Bilbao: Espasa-Calpe SA.

Gómez Martínez, V. (2007). Dinámicas de intervención y conservación en los Conjuntos Históricos en la provincia de Sevilla. Trabajo Final de Master. Sevilla: Universidad de Sevilla.

Gómez Martínez, V. et al. (2008). Dinámicas de intervención y conservación en los Conjuntos Históricos en la provincia de Sevilla. En Actas del IX Congreso internacional de rehabilitación del Patrimonio arquitectónico y edificación. Gran Canaria: Centro Internacional de Conservación del Patrimonio.

Gómez Martínez, V. (2017). La casa sin nombre. Una casa popular tradicional en la provincia de Sevilla. Tesis doctoral. Sevilla: Universidad de Sevilla.

Martín Galindo, J. L., y Miguel Orovengua, J. (2004). Arquitectura tradicional en la provincia de Badajoz. Badajoz: Departamento de Publicaciones de la Diputación de Badajoz.

Melero Melero, M. L. (1995). Inventario andaluz de arquitectura popular (2 ${ }^{a}$ fase): uso preferente habitación. Provincia de Sevilla. Catálogo fotográfico, fichas de inventario e informe final. Trabajos inéditos. Sevilla: Consejería de Cultura y Medio Ambiente. Dirección General de Bienes Culturales.

Mendoza Yusta, R. B. (2009). Arquitectura vernácula en el valle del Guadiato. Arte, arqueología e historia, 16, 481-488.

Rodríguez Becerra, S. et al. (2001). Arquitectura vernácula. En Proyecto Andalucía. Antropología. Sevilla: Publicaciones comunitarias.

Torres Balbás, L. (1930). La vivienda popular. Folklore y costumbres de España. Barcelona: Casa editorial Alberto Martín.

\section{Legislación y normativa}

Gaceta de Madrid No 145 de 25 de mayo de 1933 - Ley relativa al Patrimonio Histórico Artístico Nacional.

Boletín Oficial del Estado No 155 de 29 de junio de 1985 - Ley 16/1985, de 25 de junio, del Patrimonio Histórico Español.

Boletín Oficial de la Junta de Andalucía No 59 de 13 de julio de 1991 - Ley 1/1991, de 3 de julio, de Patrimonio Histórico de Andalucía.

Reglamento de Organización Administrativa del Patrimonio Histórico de Andalucía aprobado mediante Decreto 4/1993, de 26 de enero 
Boletín Oficial de la Junta de Andalucía № 248 de 19 de diciembre de 2007 - Ley 14/2007, de 26 de noviembre, del Patrimonio Histórico de Andalucía.

Boletín Ofícial de la provincia de Sevilla. № 187 de 13 de agosto de 2014 - Acuerdo del Pleno de fecha 29 de mayo de 2014 por el que se aprueba definitivamente el Plan Especial de Conjunto Histórico de Arahal (Sevilla). 